\title{
Digital Business Models for Peer-to-Peer Networks: Analysis and Economic Issue
}

\author{
RAMAYYA KRISHNAN \\ H. John Heinz III School of Public Policy and Management, Carnegie Mellon University \\ MICHAEL D. SMITH * \\ H. John Heinz III School of Public Policy and Management, Carnegie Mellon University \\ ZHULEI TANG \\ Krannert School of Management, Purdue University \\ RAHUL TELANG
}

H. John Heinz III School of Public Policy and Management, Carnegie Mellon University

\begin{abstract}
Peer-to-peer (P2P) services allow users to share networked resources, notably bandwidth and content, from the edges of the network. These services have been popularized because of file sharing - particularly the sharing of unlicensed copyrighted files. However, content owners are increasingly exploring the ability of peer-to-peer networks to accommodate legitimate content distribution and promotion. In this article, we review the economic characteristics of P2P networks and outline the implications of these characteristics on efforts to counteract illegal piracy and on potential uses of P2P networks in a commercial media distribution strategy.
\end{abstract}

\section{Introduction}

Peer-to-Peer (P2P) networks have generated tremendous interest recently as a way to allow distributed users to share networked resources, notably bandwidth and content, from the edges of the network. Unlike a traditional client-server architecture, nodes in a P2P network function as both clients and servers - downloading from and providing content to other peers on the network. In this way, P2P networks facilitate the direct exchange of files between users without the need for mediation by centralized servers.

\footnotetext{
* Contact Author. H. John Heinz III School of Public Policy and Management, Carnegie Mellon University, Pittsburgh, PA, 15213 E-mail: mds@cmu.edu We thank the editor of the special issue, two anonymous referees, and participants at the 2005 Economics of Digital Business Models conference for valuable comments on this research. Financial support was provided by the National Science Foundation through grants IIS-0118767 (Krishnan), IIS-0448516 (Smith), and CNS-0546009 (Telang).
} 
Although the history of P2P networks can be traced to early Internet applications, such as Usenet and DNS, P2P networks have gained particular popularity in recent years because of file sharing - notably the sharing of unlicensed copyrighted files. This illegal file sharing led media companies to initially eschew P2P networks as content distribution platforms and to resort to a series of lawsuits against these services. Even those P2P companies that were able to make a transition from illegal content distribution to "legitimate" content delivery services, such as Napster, were frequently forced by content providers to adopt a centralized client-server delivery mechanism, instead of a traditional P2P architecture.

Recently, however, media distribution companies have started to test the use of P2P networks as part of their promotion and distribution strategy. Established artists, such as Steve Winwood, have used P2P networks to distribute free copies of their songs intended to promote recently released albums (Reuters, 2004). Extending this concept, companies such as Peer Imact, INTENT Mediaworks (Young et al, 2005), Altnet (AP, 2003), SnoCap (Healey, 2004), and weed-files.com (Dean, 2004) offer media companies and artists with secure distribution of content over P2P networks and platforms to allow consumers to sample and purchase desired content. Other companies are using P2P for the distribution of digital radio (AudioFeast, Indie911), "broadcast” video (Cybersky, DAVE TV, NetCableTV, Kontiki), and independent films (Transmission Films, Cinequest Film Festival) (Schonfeld 2002). More recently, Warner Brothers Home Entertainment Group announced that they will use BitTorrent to distribute protected copies of their movies and television shows (see Marlowe, 2006).

Other companies have also incorporated P2P services into a wide-variety of business applications. For example, P2P-based sharing of compute capacity has been adopted for collaborative computing (for example, SETI@Home), instant messaging, enterprise information sharing (for example, Bad Blue), and distributed data storage (PeerioData) (Krishnan et al, 2004). Recently, there is blurring of distinction between different P2Pbased applications. For example, at one point, the Kazaa user agreement gave Kazaa's parent company, Brilliant Digital Entertainment, the right to sell extra processing power as part of their business model (Carlson, 2002).

In this article, we analyze the economic characteristics of P2P networks, and discuss how these characteristics impact their use as distribution mechanisms for both legitimate and illegitimate content. The remainder of the article proceeds as follows. In Section 2, we analyze the characteristics and history of P2P networks, highlighting why P2P networks may be important for online business models. In Section 3, we discuss the economic characteristics of P2P networks and review the relevant economic and computer science literatures on P2P networks with an emphasis on the efficiency issues that need to be addressed when P2P networks are used as part of a business model. In Section 4, we conclude that although $\mathrm{P} 2 \mathrm{P}$ networks have potential advantages over traditional distribution channels, several important issues need to be considered for $\mathrm{P} 2 \mathrm{P}$ to be used as part of a business model.

\section{Background}

As noted above, the peer-to-peer architecture has its roots in the early design of the Internet and many early Internet applications, such as the early ARPAnet backbone, and 
Domain Name Server (DNS) system, and USENET news groups. ${ }^{1}$ In each of these cases, services were provided by nodes directly to one another (for example, in USENET, messages were passed between nodes without intermediation via a central server).

While the P2P architecture was present in the early Internet, it was not until June 1999, with the release of the Napster client that P2P networks began to gain a place in the public consciousness. Initially, Napster was intended for a small group of friends to share MP3 files, because standard search engines were unreliable to locate these files and available music indexes were out of date. Within just a few days after its release, the client was downloaded by 10,000 to 15,000 people (Spencer, 2000), and by the end of the year 1999, the number of Napster users reached 1 million (Merriden, 2001). At its peak, Napster had 13 million registered users, with about 1.6 million of these users logged into the service at any one time (Romer, 2002). College students were among the first to embrace Napster. By late 1999, Napster was consuming up to $30 \%$ of the Internet bandwidth at a number of universities such as Oregon State and Florida State. In February 2000, New York University became one of the first schools to ban Napster. As the Napster community kept growing, it also drew attention from the Recording Industry Association of America (RIAA), who sued Napster for copyright violation. In July 2001, a U.S. District Court judge ordered Napster to close, which forced the company to shut down the service (Moon, 2003).

However, the demise of Napster did not diminish demand for pirated media files. If anything, the publicity surrounding the case may have increased demand for P2P filesharing services. In the years after Napster, a collection of P2P networks have gained popularity and notoriety including iMesh, OpenNap, Gnutella, Kazaa, and most recently BitTorrent. In spite of an increasing number of lawsuits against these networks and their users, P2P usage has also increased. By some estimates, BitTorrent currently accounts for one-third (Thompson, 2005) to over half (Borland, 2005) of all Internet traffic. Figure 1 shows that, according to slyck.com, the average number of users logged into P2P networks worldwide increased from 3.8 million in August 2003 to 9.7 million in January 2006 and cache-logic estimates that these online users are sharing on the order of 10 petabytes of data, which is the equivalent of about 2 million full-length DVDs or 230 million CDs.

The cost to serve 10 million customers with 10 petabytes of data would be astronomical using current technology. Yet, P2P networks accomplish this by using spare bandwidth, storage and processing cycles shared for free by its members. And herein lies one of the important advantages of P2P networks for legitimate media distribution: their ability to scale to accommodate millions of users, sharing billions of large files.

Network scalability is a function of architectural design. Prior research (Asvanund et al, 2004) has identified three classes of P2P architectures: centralized, distributed, and hybrid. Napster, iMesh, and Audiogalaxy are examples of a centralized P2P architecture, where there is a central directory storing an index of P2P content, but where the physical copy of the content still resides on individual peers. The advantage of this architecture is that query processing imposes low overhead on individual peers, because peers pass their queries directly to the central index. However, the main disadvantage of this approach is that the central server serves as both a bottleneck on query processing and a single point of failure for the network - as illustrated by the court-order shutdown of the Napster network's central servers. The BitTorrent protocol adopts a similar architecture. BitTorrent users typically locate files they are interested in by searching one of a number of "tracker

\footnotetext{
${ }^{1}$ See http://www.anu.edu.au/people/Roger.Clarke/EC/P2POview.html for a good overview.
} 
sites” that maintain a searchable index of metadata associated with various files available for download from other BitTorrent nodes.

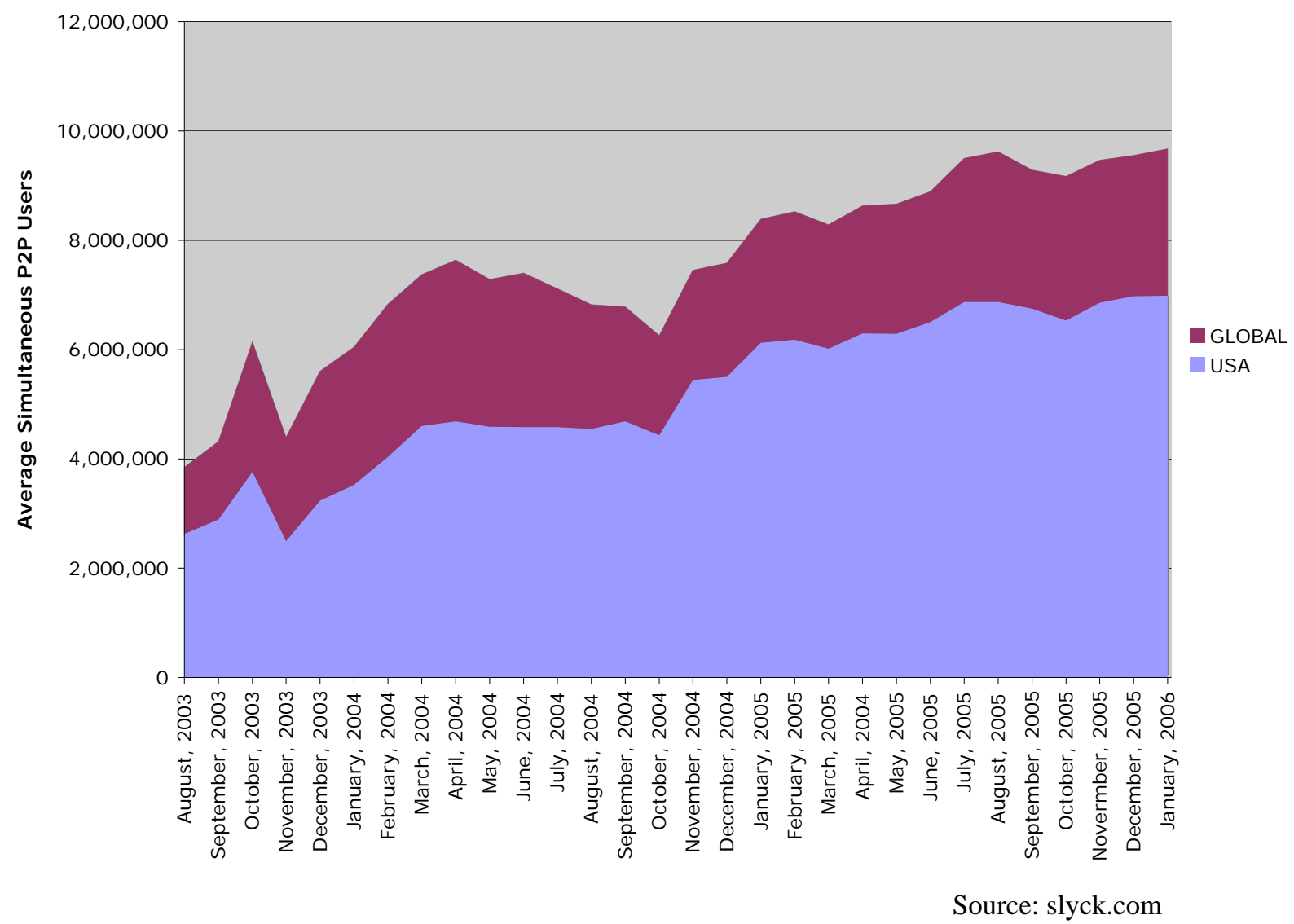

\section{Figure 1: Average simultaneous P2P users}

A second generation of $\mathrm{P} 2 \mathrm{P}$ networks sought to address concerns surrounding this single point of failure by adopting a fully decentralized architecture. For example, in the Gnutella v0.4 protocol, there is no index of content in the network. Instead, individual peers maintain connections to a small set (typically 4-5) of other peers in the network. Queries are then passed through this inter-connected mesh network, with query depth being determined by a time-to-live parameter (TTL) which is typically limited to seven or fewer hops in the network. This architecture overcame the single point of failure problem but at the expense of scalability: Query processing imposed a large externality on other members of the network, degrading network performance.

Hybrid $\mathrm{P}_{2} \mathrm{P}^{2}$ architectures seek to adopt the best of both the centralized and decentralized architectures. Examples of hybrid P2P architectures include Gnutella v0.6 and Kazaa. In the hybrid architecture, there are intermediate nodes (or ultrapeers) maintaining directories of local content among the peers they are connected to. Individual peers connect directly to these ultrapeers, and ultrapeers are connected to each other in a

\footnotetext{
${ }^{2}$ DHT (Distributed Hash Tables) provide an alternative approach to the ideas discussed in this paper. A prototypical example of a DHT that is well known is CHORD (see http://pdos.csail.mit.edu/chord/). DHTs are outside the scope of our discussion in this paper.
} 
mesh network similar to decentralized networks. Ultrapeers are chosen by the network based on their bandwidth, processing power, and stability on the network. Searches propagate from individual peers to their locally connected ultrapeer, and then through the mesh of interconnected ultrapeers, providing the network with improved query processing efficiency versus Gnutella 0.4 networks.

\section{Economics issues}

In this section, we discuss a variety of mechanisms to promote sharing in P2P networks. A number of empirical studies (Adar and Huberman, 2000; and Asvanund et al, 2004) document free riding in P2P networks and argue that high levels of free riding may result in the collapse of these networks (that is, the tragedy of the commons). This motivates the rationale for why mechanisms to promote sharing have attracted significant attention in p2p networks. We begin in Section 3.1 by comparing the economic properties of P2P networks with those of public and club goods and find a great deal of similarity between them. We then discuss specific incentive mechanisms to stimulate sharing in P2P networks. Section 3.2 studies pricing mechanisms; Section 3.3 focuses on reputation based mechanisms; Section 3.4 discusses implementation issues with a focus on digital technologies.

\subsection{Incentives, public goods, and club goods}

While differing in specific architectural design, P2P networks share some economic characteristics that are similar to public goods and club goods (Cornes and Sandler, 1996). Traditionally, goods that are not excludable in supply and are non-rival in demand are called public goods. Lighthouses are a typical example of public goods. Club goods are excludable in supply (that is, to club members) but usually non-rival in demand, although an individual's consumption may depend on the number of other persons with whom he must share his benefits (Buchanan, 1965). Examples of club goods include country clubs, and cable TV broadcasts. The purpose of clubs is to exploit economies of scale and to share public goods. There are numerous papers on the efficiency of clubs for facilitating the sharing of public goods in traditional environments (Sandler and Tschirhart, 1980). Among them, Berglas (1976) established a result regarding the competitive provision of club goods, which states that club theory does not necessarily exclude efficient provision by market. Helsley and Strange (1991) examined the competitive provision of club goods with costly exclusion and considered two alternative exclusion regimes: fine and coarse exclusion. With fine exclusion, a provider can charge both a membership fee and a per use price. With coarse exclusion, a provider can charge a membership fee only. This solution suggests that pricing mechanisms may also play a role in designing efficient P2P networks, as we discuss in Section 3.2 below.

We find several notable similarities between public and club goods, and the manner in which resources are shared in $\mathrm{P} 2 \mathrm{P}$ networks. Specifically, goods provided in P2P networks are typically non-excludable in supply in the sense that once they are made available on the network, they are made available to all network users. Moreover, in an ideal case, P2P goods can be thought of as non-rival in demand in the sense that when one peer downloads a file, they also share that file back with the rest of the network. However, two factors can increase rivalry among users. First, downloading over an asymmetric connection (one 
where the download speed is several orders of magnitude greater than the upload speed) may cause rivalry through a net reduction in download bandwidth available to other peers. More importantly, however, free-riding (where a peer consumes network resources without sharing their own resources in return) may also increase rivalry by reducing the available supply of content. Goods provided in P2P networks can also be thought of as excludable in supply if they are provided on a priority or exclusive basis to members of a local group of interconnected peers (Asvanund et al, 2003).

Recently, a variety of mechanisms have been proposed to share resources in P2P networks. In light of the similarities between P2P network provision and public and club goods, many of the proposed mechanisms can be seen as assuring adequate provision of public or club goods. We review these mechanisms and differentiate them in terms of how incentives are provided to promote sharing and how the mechanisms are implemented using digital technologies.

\subsection{Pricing mechanisms in P2P networks}

As noted earlier, the most fundamental question in P2P networks is how to stimulate peers' incentives to cooperate or contribute resources in P2P networks (Bredin, 2004) - a problem that is also common to public and club goods settings. As discussed above, this problem is directly related to the nature of these networks. In most P2P protocols, peers can download without sharing content/bandwidth in return; and in many protocols, the network does not impose a penalty on the free-riding peer. Moreover, when free-riding is high, all nodes in the network are subject to congestion.

The problem of free-riding has been studied widely in the economic literature (Lindahl 1919; Samuelson 1954, 1955, 1969; Olson, 1965; Hardin, 1968; Clarke, 1971; Groves, 1973; Groves and Ledyard, 1987; Palfrey and Rosenthal, 1984; and Cornes and Sandler, 1996). Numerous mechanisms have been proposed to address the free-rider problem in public goods provision. In the public goods setting, the failure of each consumer to consider the benefits for others of her public good provision is known as the free-rider problem, or following Hardin (1968) as simply "the tragedy of the commons". It is also well known that the free-rider problem may worsen as group size increases (Olson, 1965).

Free-rider problems result in an inefficient level of provision of public goods. It is also seen empirically in P2P networks where sharing levels are frequently below the social optimal level (Krishnan et al, 2004). Another companion problem is that peers often fail to consider the impact of their consumption of network resources on other users. For example, if one user sends too many download requests, the responding node may be too busy to process the requests, therefore, unable to handle other users' requests. This phenomenon is often referred to as an externality, specifically in this context, consumption externality. That is, the utility of one consumer is directly affected by the actions of another consumer.

However, many of the mechanisms aimed at alleviating the free-rider problem share one undesirable property: participants in the mechanisms do not have freedom not to participate. This raises the question of what will happen if voluntary participation is allowed. Palfrey and Rosenthal (1984) analyzed the provision of a binary public good with voluntary contributions. The private provision of discrete public goods (Bagnoli and Lipman, 1989) is of particular interest to P2P research because it assumes voluntary contributions by individual player - a feature that typical P2P networks also possess. 
In P2P networks, where content can possibly be downloaded and consumed irrespective of the level of contribution, the good is provided only if a sufficient (threshold) number of voluntary contributions are made. For this reason, the problem is also known as "voluntary contribution threshold games" (van de Kragt, Orbell, and Dawes, 1983; and Bliss and Nalebuff, 1984). In these games, if the threshold is not met, that is if the total contributions do not meet or exceed the threshold, all contributions are returned and the public good is not provided. When each individual has incomplete information about certain characteristics of other individuals, for example, the cost of contribution, the value of the public good, or the "altruism" component, the asymmetry underlying the incomplete information will explain why some of the players will contribute while others will not. In the private contribution game, public goods can either be overprovided (Gradstein and Nitzan, 1990) or underprovided (Nitzan and Romano, 1990).

Various mechanisms have been studied in the context of public goods to achieve efficient provision. All bear the same idea - to endogenize the externality that consumers fail to consider. The Lindahl equilibria (Lindahl, 1919) is an example of such a mechanism incorporating a market institution. Under this mechanism, one market is created for each consumer's consumption of public goods. Prices can be different across markets. The equilibrium outcome of the public good consumption by each consumer is exactly the efficient level. An efficient mechanism does not necessarily have other desirable properties. For example, Groves and Ledyard (1977) proposed a mechanism that achieves a Pareto efficient allocation, but it does not satisfy individual rationality (Saijo and Yamoto, 1997). Subsequently, a variety of mechanisms have been proposed satisfying both Pareto efficiency and individual rationality, as well as other desirable properties such as individual feasibility and balancedness (Groves and Ledyard, 1987; and Hurwicz, Maskin, and Postlewaite, 1995).

The incentive problem in P2P networks poses an obvious obstacle for P2P to be used as a distribution channel. Lacking incentives, free-riding will likely abound. There is recent growth of $\mathrm{P} 2 \mathrm{P}$ literature from the computer science and economics perspective that address this problem. The most common solution is using explicit or implicit pricing to endogenize the externality that peers impose on the network. Pricing mechanisms can often be used to deal with inefficient provision of public goods (Coase, 1960; and Varian, 1989); and not surprisingly, pricing mechanisms have also been proposed in the context of P2P networks. In Adler et al (2004), each contributing peer offers a different price, while a downloading peer selects from these peers on the basis of the offered prices. Tamilmani, Pai, and Mohr (2004) examine a trading model in which peers use a pairwise bandwidthbased currency to reconcile trading differences with each other. Significantly, in their mechanism, peers who contribute to the network receive a higher benefit (that is, download speed) than free-riding peers do. Similarly, Golle, Leyton-Brown, and Mironov (2001) and Chandan and Hogenborn (2001) show that micropayment based systems can be used to mitigate the free-riding problem in P2P networks, and Gupta and Somani (2004) examine architecture for pricing and sharing of computing resources in P2P networks. While each of the above mechanisms mitigates congestion in P2P networks, they do not guarantee efficiency as an equilibrium outcome. For example, Chandan and Hogenborn (2001) point out that even with pricing and perfect competition, congestion externality still exists, so peering organized through a club may be the best solution.

While most of the research studying the free-riding problem in P2P networks focuses on direct and explicit incentives to encourage peers to share, Krishnan et al (2004) analyze 
the possibility that users may share their content based entirely on self-interest. The intuition is that sharing will draw traffic away from other peers in the network to the sharing peer, thereby increasing the chance that the sharing peer will be able to get her desired content from other peers on the network. Thus, it is possible for a peer to increase her private utility through sharing. They propose to differentiate the quality of service provided to peers based on whether they share content. Degrading the quality of service can be seen as implicit pricing mechanisms. Significantly, they also find that it may not be socially optimal for all users to share their content, because the sharing cost is not justified by the potential benefit.

BitTorrent is a real world example of building incentives into protocol design (Cohen, 2003). In a way, it uses implicit pricing mechanism. "Prices" are automatically enforced, because when multiple people are downloading the same file at the same time, they upload pieces of the file to each other. The basic idea is to make a peer's download rate proportional with its upload rate.

Pricing based mechanisms refer more generally to taxes or quotas as a way to regulate the access to public goods. In practice, the degree of externality is only known to a user, therefore, asymmetry of information can make pricing difficult. A natural question is whether there exist other mechanisms that do not involve pricing but can still stimulate individuals to contribute to $\mathrm{P} 2 \mathrm{P}$ networks. One solution is through reputation based mechanisms, which we discuss in Section 3.3.

\subsection{Reputation based mechanisms in P2P networks}

Reputation based systems track a user's contribution over a longer time period (Krishnan, Smith, and Telang 2003). The basic idea is a tit-for-tat strategy. Vishnumurthy, Chandrakumar, and Sirer (2003) propose KARMA to track each user's contribution and consumption. The "karma" score is used to prioritize peers who have high contribution. Another feature of reputation based mechanism is that they are built on the premise that peers have repeated interactions - a characteristic that tends to distinguish P2P networks from public goods. Therefore, some peers may choose to contribute in some periods, while in other periods, they free-ride. Meanwhile, duplication of the content will exist with the passage of time. The dynamic provision of public goods provides a partial solution to peers incentives to contribute in a repeated setting (Gradstein and Nitzan, 1990; Gradstein ,1992; and Konrad, 1994). When incorporating time dynamics and incomplete information, public goods may be underprovided due to a delay in the provision. Not only time dynamics can change the equilibria; when players decide to contribute in more than one period, their incentives are likely to change. Sometimes, sequential contribution may even exacerbate the free-rider problem (Varian, 1994).

Reputation systems have been studied extensively for electronic commerce sites (for example, Dellarocas, 2003). Comparable to eBay's reputation mechanisms, reputation mechanisms in $\mathrm{P} 2 \mathrm{P}$ environments need to consider repeated interaction among users. Unlike the eBay reputation mechanism, however, reputation mechanisms in P2P networks require more rapid information transmission and updating. Designing a viable reputation mechanism in P2P environments requires a consideration of both issues.

Morselli, Katz, and Bhattacharjee (2004) analyze the robustness of a reputation-based protocol, where a user who free-rides will be punished to the extent that others refuse to share resources with her. The game-theoretic reputation models used in this and other similar papers have one desired property, that is, if the reputation system is designed 
properly, trust should be the outcome of the equilibria of a repeated game (Despotovic and Aberer, 2004). Hwang and Lee (2004) analyze an Eigenvector-based reputation system (Kamvar, Schlosser, and Garcia-Molina, 2003) with differentiated admission using evolutionary game theory. Peers have more incentives to contribute because of access priority. Their mechanism also allows the transaction of reputation score among agents over time. Lai et al (2003) use the evolutionary prisoners' dilemma to study reputationbased mechanisms and find the prisoners' dilemma maybe overturned. Wang and Vassileva (2003) propose a Bayesian network-based trust model for building reputation in P2P networks. Peers can update their trust in other peers based on their repeated interactions.

Since P2P networks share some characteristics with club goods as noted above, the design of a reputation mechanism can also exploit this feature. Abrams, McGrew, and Plotkins (2004) propose an EigenTrust-based reputation system, in which peers are partitioned into groups and incentives are structured so that a peer only downloads from peers in one particular peer group. They demonstrate that the total error in trust values decreases with the number of groups. Similarly Asvanund et al (2003) propose a file sharing architecture in which a Gnutella ultrapeer and its local network of leaf nodes form a "club." By simulating the performance of the architecture, they show that the club model can organize self-interest peers into local clubs and increase peers' incentives to share content at the same time.

Despite the promise of using reputation and trust to promote sharing in P2P networks, a drawback of the reputation system solution is that systems can deteriorate by the spread of false reputation ratings. Buchegger and Le Boudec (2004) propose a system that can avoid this problem. In this system, everyone maintains two ratings: one rating is about others' reputation; the other rating is about how honest the others are in the reputation system. Over time, reputation ratings are modified by accepted information using a Bayesian approach. Trust ratings are updated based on the compatibility of second hand reputation information with prior reputation ratings. This approach is robust against false ratings and efficient at detecting misbehavior.

These reputation based models provide a viable tool to better manage P2P networks. However, the following issues need to be addressed before implementing these models in P2P networks. First, because the identity of peers is dynamically changing, mechanisms need to incorporate systems to keep track of peers. Second, the administration of the evaluation system in a fully decentralized P2P network presents additional challenges related to synchronizing identities (Krishnan, Smith, and Telang, 2003).

Interestingly, the literature notes that pricing - and reputation - based mechanisms can complement each other. Ranganathan et al (2003) compare reputation and pricing-based mechanisms and find these incentive schemes can be used together to counter selfish user behavior. Without incentive mechanisms, no contribution is an equilibrium, but this equilibrium is inefficient since users could have obtained higher payoffs had they all made the opposite choice. This result is reminiscent of prisoners' dilemma.

In summary, in order to use $\mathrm{P} 2 \mathrm{P}$ networks as a distribution channel, one should consider its similarity to public goods or club goods. However, the unique features of P2P networks also need to be taken into account. For example, the size of the endowment of resources in a P2P network varies based on how many peers contribute (Krishnan et al, 2004), which means that each peer who shares resources increases the size of the endowment to all other members. Peers have dual roles of being consumers of the P2P 
resources and providers as well. Over time, the same content can be distributed to many peers, which can potentially alter the quality and quantity of available resources and thereby the dynamics of user participation.

\subsection{Implementation of sharing mechanisms}

Though the underlying principle of the $\mathrm{P} 2 \mathrm{P}$ protocol is to stimulate sharing of network resources, implementing these protocols can be a nontrivial task. Digital technologies have made it easier to manage complex mechanisms, punish non-contributors, reward contributors, and manage negative externalities. For example, in Kung and Wu (2003), in order to implement a reputation-based P2P admission system, a distributed eigenvectorbased method is used to compute user reputation, and a sampling heuristic is used to reduce computation and communication costs. Similarly, Tamilmani, Pai, and Mohr (2004) have added SWIFT, a credit-based trading mechanism to the official BitTorrent experimental client and demonstrated its ability to facilitate fairly large-scale file distribution.

Other mechanisms, such as the one proposed by Krishnan et al (2004), are based on system-wide rules differentiating the quality of service provided to peers based on the value the peer provides to the network. Sometimes, the successful implementation of the mechanisms relies not only on software protocols but also on the members of community explicitly enforcing common rules. Dutta et al (2003) investigate such a mechanism, in which the design of an explicit rating system relies on the truthful rating of community members. Klaas (2004), using a game-theoretic model, shows that in file sharing networks, groups of users can self-regulate their network usage if the users are reputation-motivated. Under certain circumstances, it may be more effective to foster communication among users rather than through formal regulations. However, this type of self-governance can fail on a system-wide level, because members may be partitioned into disjoint communities.

It is also important to note that, from the perspective of hybrid P2P architectures, designing and implementing protocols to solve the free-rider problem requires different incentive schemes for ultrapeers and leaf nodes. For example, ultrapeers handle much larger traffic than leaf nodes and are selected by the protocol. Therefore, the protocol has to provide a different set of incentives for ultrapeers. Singh et al (2003) present such a mechanism for several actors to deploy ultrapeers and to increase the effectiveness of ultrapeers. The mechanism, which functions by caching metadata and using more efficient topic-based search optimization at ultrapeers, has the potential to improve the search performance of an ultra peer's client without adding significant burden to the ultrapeer itself. Similarly, the information retrieval-based utility model proposed by Asvanund et al (2003) covers decisions made by leaf nodes and ultrapeers separately: leaf nodes determine which ultrapeer to connect to; ultrapeers choose how to manage its local community and to which other ultrapeers they should connect to.

The implementation of a specific sharing mechanism can be further complicated by the problem of pollution. Pollution seeks to deposit tampered files into P2P file sharing networks. When these files are downloaded by unaware users, they are spread into the network, causing an overall degradation of the quality of service provided by the network (Liang et al, 2005). Two forms of pollution exist: content pollution and metadata pollution. Content pollution alters the original digital content by replacing all or part of the content with white noise, cutting the duration, or inserting other irrelevant content. Metadata 
pollution changes the metadata instead of the content, thereby making it difficult for users to locate the right content. No matter what form of pollution is used, the goal of the polluter is simple: proliferate P2P networks with useless content, so that they can no longer be used to distribute problematic (for example, unlicensed copyrighted) materials for free.

Although P2P networks greatly facilitate the sharing of resources among Internet users, whether the shared content is legitimate or not, the sharing of copyrighted content raises serious concerns on the copyright holders, such as RIAA and MPAA. Forrester research estimates that the music industry lost over $\$ 700$ million in CD sales in 2003 due to sharing of copyrighted songs in P2P networks (Bernoff et al, 2003). Copyright holders, therefore, have a very different goal. Instead of promoting sharing and efficiency of P2P networks, they aim to shut down the file sharing networks. In addition to the legal efforts that the RIAA and MPAA have taken, one technique that is particularly prevalent is pollution or poisoning.

There have been a number of studies on the nature and magnitude of pollution in P2P networks. For example, Liang et al (2005) develop a measure of pollution in the FastTrack P2P networks based on the number of unique versions and copies available for popular audio content and find that pollution is pervasive for recent popular songs. The content rating mechanism of $\mathrm{KaZaA}$ is incapable of dealing with frequently introduced polluted versions of a particular song. Similarly, it is found that in the Spring of 2005, pollution was highly prevalent in the FastTrack and eDonkey systems, with as many as 50 percent of copies of popular titles being polluted (Liang et al, 2006). Pouwelse et al (2005) draw a different conclusion for the integrity in the BitTorrent/Suprnova network. Because a direct measurement of polluted files in the BitTorrent networks is difficult, they try to create a measure by inserting fake files into the network. They find that the system of moderators is effective in removing polluted content. This finding seems to be consistent with the discussion on P2P message boards, which suggests that BitTorrent/Suprnova is virtually immune to pollution. Nevertheless, BitTorrent networks are subject to denial of service attacks on trackers. Kumar et al (2006) also examine how the injection of multiple versions of decoy content impacts a user's ability to receive a valid copy. Their models incorporate user behaviors such as preferences for popular versions and abandonment after repeated failure to download a good version. They find that users are better off selecting a version without considering its popularity because the content that is very popular and spreads quickly is very likely to be a polluted version.

Given that pollution is a prevalent phenomenon in P2P file sharing networks, a variety of mechanisms have been proposed to deal with the pollution attacks in P2P systems. Liang et al (2005) have described several anti-pollution mechanisms. Some of the mechanisms require downloading a file before detecting pollution. For example, in a matching mechanism, downloaded files are compared with authentic content stored in a trusted database. In a user filtering mechanism, users need to check the integrity of downloaded files before letting others copy them. Interestingly, some mechanisms do not need downloading to detect a polluted content. Blacklist is one example, where sources that frequently inject polluted content are blocked.

Another mechanism to prevent pollution is to use reputation and trust. Similar to the reputation and trust mechanisms used to encourage sharing of content, the reputation mechanism in the context of detecting pollution relies on rating one peer by others. However, the latter reputation mechanism would rate objects, rather than peers. Walsh and Sirer (2005) propose a decentralized system for evaluating the reputation of objects. The 
system employs a network-wide voting scheme, where users evaluate objects. Evaluation from users can be weighted using the correlation between different peer opinions. Liang et al (2005) also discuss anti-pollution mechanisms using reputation and trust. For example, users may only trust friends who have provided them with "clean" content before. This idea can also be generalized to the whole P2P network, so that a user may download from the friends of his trusted friends. Despite several anti-pollution mechanisms proposed, all have their drawbacks. For example, the downloading-based mechanism demands a trusted database to maintain authenticated copies of content, which not only has a maintenance cost but could itself be the target of an attack. On the other hand, the design of a particular reputation system may be subject to deceptive ratings and high complexity of implementation.

\section{Discussion}

For the foreseeable future, P2P networks will play a critical role in the business models of the firms distributing digital content. P2P networks will pose challenges for established firms in several ways.

First, presence of P2P networks raises an important question for firms: under what circumstances does piracy cannibalizes product sales and under what circumstances may piracy actually enhance sales? Several papers in the literature note that piracy need not necessarily damage overall media sales. For example, piracy can enhance media sales by establishing an initial user base and speeding diffusion (Prasad and Mahajan, 2003), by providing free samples and increasing awareness (Peitz and Waelbroeck, 2003), and by changing the competitive pricing decision of monopolists ( $\mathrm{Gu}$ and Mahajan, 2004). It is important to note these explanations rely on imperfect consumer information or on the pirate providing a positive externality to subsequent purchasers, thus they may be more applicable to some digital products than others, a topic we discuss in more detail below. In the absence of either of these effects, piracy would seem to directly harm subsequent media sales. These differences may explain the mixed empirical results regarding the impact of piracy, with some empirical papers finding direct harm from piracy (for example, Liebowitz, 2005; and Rob and Waldfogel, 2006), others that piracy can help less popular products (for example, Bhattacharjee et al, 2006; and Blackburn, 2004), and still other papers finding no impact of piracy (for example, Oberholzer and Strumpf, 2004; and Smith and Telang, 2007).

On the other hand, P2P networks also provide media companies and artists with a new, low cost, tool for legitimate distribution that can be used for competitive advantage. For example, P2P networks have made it easier for small artists and minor labels to market and promote their albums. Typically, marketing an album is both costly and risky - and historically, only a few large record companies have had the necessary resources to market new albums. However, P2P networks and other Internet-enabled marketing channels may provide artists an opportunity to spread the word about their music at a significantly lower cost. A recent study by Bhattacharjee et al (2006) finds that minor labels have directly benefited from file-sharing networks. Thus, P2P networks may reduce the concentration in media market by lowering entry costs of promotions and advertising.

P2P networks have also made it possible for users to browse and search niche artists and albums which may not be readily available in record stores (for example, a typical 
large Tower Records brick-and-mortar store will stock less than $6 \%$ of the CDs in circulation). Such a phenomenon has already been observed in books and apparel categories (Brynjolfsson, $\mathrm{Hu}$, and Smith, 2003; and Bryjolfsson, $\mathrm{Hu}$, and Simester, 2005). Increased information availability allows users to discover specialized and niche products that meet their preferences better than more "mainstream" products. Again, this may change the competitive dynamics between well-known and less well-known artists.

P2P networks will not only impact the music industry; other digital content, such as ebooks, software, video files can also be distributed on P2P networks. P2P networks hold promise for video files as a way to reduce the significant technological costs of distributing large video files from a central server. In contrast to central server architectures, P2P networks exhibit remarkable scalability (more than 3.5 million users log into P2P network at a given time). This scalability has made P2P networks attractive for a variety of high bandwidth applications such as digital radio (AudioFeast, Indie911), broadcast video (cybersky, DAVE TV, etc.), independent films (Cinequest Film festival downloads over BitTorrent), and independent music (Altnet and weed files for licensed content distribution).

In some categories, P2P networks may even change industry perceptions of the impact of piracy. For example, King and Lampe (2002) analyze the profitability of software piracy theoretically and find that piracy will only be profitable if the ability to pirate is inversely correlated with customer willingness-to-pay and when a producer cannot price discriminate between potential pirates and other customers. The intuition is that because of network externalities in the market for software, allowing some customers with low willingness-to-pay to pirate increases the customer base, therefore increasing the willingness-to-pay of non-pirates and resulting in higher profits overall. The result, to some extent, justifies firms' incentives to use P2P network as a potential business model for distributing digital content.

However, one should generalize these findings to other categories of digital goods with some caution. While different categories of digital content share similar features, such as a zero marginal cost of production, they differ in terms of appropriability (Besen and Kirby, 1989), the importance of network externalities, and the informational role of free copies (Peitz and Waelbroeck, 2006).

For example, network externalities may be significantly more important for software than they are for other digital content categories such as music. The information role of free copies may also differ across product categories based on differences in the ability to degrade the quality of the free copy, the importance of post-sale support and the importance of extra features provided with the full version of the product. Together, the differences across product categories seem to suggest that, despite the similar features shared by different forms of digital content, designing business models for the distribution of digital content needs to exploit the unique features of specific content.

Together, our analysis suggests that $\mathrm{P} 2 \mathrm{P}$ networks could become the significant components of the digital strategy for content providers in a variety of sectors. For many of these business models to succeed, we need to enhance our understanding of both the underlying technology and the relevant business models. For example, how can copyright holders use digital rights management (DRM) techniques to complement P2P technology? How can online communities complement P2P distribution models? How can security and authentication be assured in a distributed distribution environment? 
Academic research in the fields of computer science, Information Technology, and economics are helping P2P networks to become more efficient, user friendly and ubiquitous, even as the business community is taking notice of the possible uses of these networks. Going forward, there are several exciting research avenues for social scientists and economists. To achieve these goals, there is a need for rigorous theoretical and empirical work that would provide deeper insights into how P2P networks are utilized and how they influence the market, market structure and underlying business models.

\section{$5 \quad$ References}

Abrams Z., R. McGrew, S. Plotkins (2004) "Keeping Peers Honest in EigenTrust," Working Paper, Second Workshop on Economics of Peer to Peer Network, Stanford University.

Adar, E. and Huberman, B. A. (2000) "Free riding on Gnutella," First Monday, 5. http://www.firstmonday.dk/issues/issue5_10/adar/

Adler, M., R. Kumar, K. Ross, D. Rubenstein, D. Turner, D. D. Yao. (2004) “Optimal Peer Selection in a Free-Market Peer-Resource Economy,” Working Paper, Second Workshop on Economics of Peer to Peer Network, University of Massachusetts Amherst.

Associated Press (2003) "Kazaa Looks to Legitimate Arm for Salvation,” February 3. http://www.usatoday.com/tech/news/2003-02-03-kazaa_x.htm

Asvanund, A., K. Clay, R. Krishnan, M.D. Smith (2004) "An Empirical Analysis of Network Externalities in Peer-To-Peer Music Sharing Networks," Information Systems Research, 15: 155-174.

Asvanund, A., R. Krishnan, M. Smith, R. Telang (2003) "Intelligent Club Management in Peer-to-Peer Networks,” Working Paper, Carnegie Mellon University.

Bagnoli, M. and Lipman, B.L (1989) "Provision of Public Goods: Fully Implementing the Core through Private Contributions,” The Review of Economic Studies, 56: 583-601.

Berglas, E. (1976) “On The Theory of Clubs,” American Economic Review, 66: 116-21.

Bernoff J., C. Charron, A. Lonian, C. Q. Strohm, G. N. Flemming (2003) "From Discs to Downloads," Forrester Research, Inc.

Besen, S.M. and Kirby, S.N (1989) "Private Copying, Appropriability, and Optimal Copying Royalties,” Journal of Law and Economics, 32: 255-280.

Bhattacharjee S., R. Gopal, K. Lertwachara, J. Marsden, R. Telang (2006) “The Effect of Digital Sharing Technologies on Music Markets: A Survival Analysis of Albums on Ranking Charts,” forthcoming, Management Science. 
Blackburn, D. (2004) “Online Piracy and Recorded Music Sales,” Working Paper, Harvard University.

Bliss, C. and Nalebuff, B. (1984) "Dragon-slaying and Ball-room Dancing: The Private Supply of a Public Good,” Journal of Public Economics, 25: 1-12.

Borland, J. (2005) "P2P Users Traveling by eDonkey," CNET News, August 28. http://news.com.com/2100-1025_3-5843859.html

Bredin, J. (2004) "Strategic Negotiation of Bandwidth in Cooperative Networks," ICEIS 2004, Proceedings of the 6th International Conference on Enterprise Information Systems, Porto, Portugal, April 14-17.

Brynjolfsson, E., Y. Hu, M.D. Smith (2003) "Consumer Surplus in the Digital Economy: Estimating the Value of Increased Product Variety,” Management Science, 49: 1580-1596.

Brynjolfsson, E., Hu J., and, D. Simester (2005) "Goodbye Pareto Principle, Hello Long Tail: Modeling and Measuring the Effect of Search Cost on Product Sales Distribution," Working Paper, Massachusetts Institute of Technology.

Buchegger, S., J. Le Boudec (2004) “A Robust Reputation System for P2P and Mobile Adhoc Networks,” Working Paper, Second Workshop on Economics of Peer to Peer Network, EPFL-IC-LCA.

Buchanan, J.M. (1965) “An Economic Theory of Clubs,” Economica, 32: 1-14.

Carlson, S. (2002) “KaZaA's Sneaky Software Worries Colleges,” Chronicle of Higher Education, April 26.

Chandan, S., Hogendorn C. (2001) "The Bucket Brigade: Pricing and Network Externalities in Peer-to-Peer Communications Networks,” Telecommunications Policy Research Conference, Alexandria, VA, October 27-29.

Clarke, E. H. (1971) “Multipart Pricing of Public Goods,” Public Choice, 11: 17-33.

Coase, R. (1960) “The Problem of Social Cost,” Journal of Law and Economics, 3: 1-44.

Cohen, B. (2003) "Incentives Build Robustness in BitTorrent," First Workshop on the Economics of Peer-to-Peer Systems, University of Berkeley.

Cornes, R. C. and T. Sandler (1996) The Theory of Externalities, Public Goods, and Club Goods. Cambridge University Press: New York.

Cox, B. (1996) Superdistribution: Objects as Property on the Electronic Frontier. Addison-Wesley: Reading, MA.

Dean, K. (2004) “File Sharing Growing Like a Weed,” Wired News, November 22.

Dellarocas, C. (2003) "The Digitalization of Word-of-Mouth: Promise and Challenges of Online Reputation Mechanism,” Management Science, 49: 1407-1424. 
Despotovic, Z., K. Aberer (2004) “Maximum Likelihood Estimation of Peers' Performance in P2P Networks," Working Paper, Second Workshop on Economics of Peer to Peer Network, Harvard University.

Dutta, D., A. Goel, R. Govindan, H. Zhang (2003) "The Design of A Distributed Rating Scheme for Peer-to-Peer Systems,” Workshop on Economics of Peer to Peer Network, Working Paper, University of Southern California.

Golle, P., K. Leyton-Brown, I. Mironov (2001) "Incentives for Sharing in Peer-to-Peer Networks,” Proceedings of the 2001 ACM Conference on Electronic Commerce, Tampa.

Groves, T. (1973) “Incentives in Teams,” Econometrica, 41: 617-31.

Groves, T., J. Ledyard (1977) “Optimal Allocation of Public Goods: A Solution to The Free rider Problem,” Econometrica, 45: 783-810.

Groves, T., J. Ledyard (1987) “Incentive Compatibility Since 1972,”in (ed.), Information, Incentives, and Economic Mechanisms: Essays in Honor of Leonid Hurwicz, University of Minnesota Press: Minneapolis.

Gradstein, M. (1992) “Time Dynamics and Incomplete Information in the Private Provision of Public Goods,” Journal of Political Economy, 100: 581-97.

Grandstein, M., S. Nitzan, S. (1990) "Binary Participation and Incremental Provision of Public Goods,” Social Choice and Welfare, 7: 171-92.

Gu, B., V. Mahajan (2004) “The Benefits of Piracy: A Competitive Perspective,” Sixteenth Workshop on Information Systems and Economics, Washington, DC.

Gupta, R., A.K. Somani (2004) "CompuP2P: An Architecture for Sharing of Computing Resources In Peer-to-Peer Networks with Selfish Nodes," Second Workshop on Economics of Peer to Peer Network, Harvard University.

Hardin, G. (1968) “The Tragedy of the Commons,” Science, 162: 1234-48.

Healey, J. (2004) “Music Industry Taking Cues From File Sharing,” Los Angeles Times, July 26.

Helsley, R.W., W.C. Strange (1991) "Exclusion and the Theory of Clubs," Canadian Journal of Economics, 24: 888-899.

Hurwicz, L., E. Maskin, A. Postlewaite (1995) "Feasible Implementation of Social Choice Correspondences by Nash Equilibria," Essays in Honor of Stanley Reiter, Kluwer Academic Publishers: Boston.

Hwang, J., C. Lee (2004) “Agent-based Modeling for Differentiated Admission in P2P Systems Using Evolutionary Game Theory Focused on Ownership Reputation,” Working Paper, Second Workshop on Economics of Peer to Peer Network, Seoul National University. 
Kamvar, S, M.T. Schlosser, H. Garcia-Molina (2003) "The EigenTrust Algorithm for Reputation Management in P2P Networks," Proceedings of the 12th International Conference on World Wide Web, Budapest, Hungary.

King, S. P., R. Lampe (2002) "Network Externalities and the Myth of Profitable Piracy," Working Paper, Intellectual Property Institute of Australia (IPRIA).

Klass, M. (2004) “Over-contribution in Discretionary Databases,” Working Paper, Second Workshop on Economics of Peer to Peer Network, University of British Columbia.

Konrad, K.A. (1994) "The Strategic Advantage of Being Poor: Private and Public Provision of Public Goods,” Economica, 61: 79-92.

Krishnan, R., M.D. Smith, R. Telang (2003) “The Economics of Peer-to-Peer Networks,” Journal of Information Technology Theory and Applications, 5: 31-44.

Krishnan, R., M. Smith, Z. Tang, R. Telang (2004) “The Virtual Commons: Understanding Content Provision in Peer-to-Peer File Sharing Networks,” Working Paper, Carnegie Mellon University.

Kumar, R., D. Yao, A. Bagchi, K.W. Ross, D. Rubenstein (2006) "Fluid Modeling of Pollution Proliferation in P2P Networks,” ACM SIGMETRICS Performance Evaluation Review, 34(1): 335-346.

Kung, H. T., C. Wu (2003) "Differentiated Admission for Peer-to-Peer Systems: Incentivizing Peers to Contribute Their Resources,” Working Paper, Workshop on Economics of Peer to Peer Network, Harvard University.

Lai, K., M. Feldman, I. Stoica, J. Chuang (2003) "Incentives for Sharing in P2P Networks,” Working Paper, Workshop on Economics of Peer to Peer Network, University of California at Berkeley.

Liang J. R. Kumar, Y. Xi, K.W. Ross (2006) "The Index Poisoning Attack in P2P FileSharing Systems,” Proceedings of IEEE INFOCOM, Barcelona, Spain.

Liang J. R. Kumar, Y. Xi, K.W. Ross (2005) "Pollution in P2P File Sharing Systems," Proceedings of IEEE INFOCOM 2005, Miami, FL.

Liebowitz, S. (2005) “Testing File-Sharing's Impact by Examining Record Sales in Cities,” Working Paper, University of Texas.

Lindahl, E. (1919) “Just Taxation - A Positive Solution,” in R. Musgrave \& A. Peacock (ed.), Classics in the Theory of Public Finance, Macmillan: London.

Marlowe, C. (2006) “Warner’s Rolling with BitTorrent,” The Hollywood Reporter, May 9. http://www.hollywoodreporter.com/thr/article_display.jsp?vnu_content_id=1002464855

Merriden, T. (2001) Irresistible Forces: The Business Legacy of Napster \& The Growth of The Underground Internet. Capstone Publishing: Oxford. 
Moon, Y. (2003) “Online Music Distribution in a Post-Napster World,” Case 9-502-093, Harvard Business School.

Moreton, T., Twigg, A. (2003) “Trading in Trust, Tokens, and Stamps,” Working Paper, Workshop on Economics of Peer to Peer Network, Cambridge University.

Morselli, R., J. Katz, B. Bhattacharjee (2004) “A Game-Theoretic Framework for Analyzingm Trust-Inference Protocols,” Working Paper, Second Workshop on Economics of Peer to Peer Network, University of Maryland.

Nitzman, S., R.E. Romano (1990) "Private Provision of a Discrete Public Good with Uncertain Cost,” Journal of Public Economics, 42: 357-70.

Oberholzer F., K. Strumpf (2004) “The Effect of File Sharing on Record Sales: An Empirical Analysis,” Working Paper, Harvard University.

Olson, M., Jr. (1965) “The Logic of Collective Action: Public Goods and the Theory of Groups,” Harvard University Press: Cambridge.

Palfrey, T., H. Rosenthal (1984) "Participation and the Provision of Discrete Public Goods: A Strategic Analysis,” Journal of Public Economics, 24: 171-193.

Peitz, M., P. Waelbroeck (2006) "Piracy of Digital Products: A Critical Review of the Economics Literature,” Information Economics and Policy, 18: 449-476.

Pouwelse, J.A., P. Garbacki, D.H.J. Epema, H.J. Sips (2005) "The Bittorrent P2P Filesharing System: Measurements and Analysis," Proceedings of the 54th International Workshop on Peer-to-Peer Systems (IPTPS’05), Ithaca.

Prasad, A., V. Mahajan (2003) "How Many Pirates Should a Software Firm Tolerate? An Analysis of Piracy Protection on the Diffusion of Software," International Journal of Research in Marketing, 20: 337-353.

Ranganathan, K., M. Ripeanu, A. Sarin, I. Foster (2003) "To Share or Not to Share: An Analysis of Incentives to Contribute in Collaborative File Sharing Environments," Working Paper, Workshop on Economics of Peer to Peer Networks, University of Chicago.

Reuters (2004) "Promo Uses P2P Networks to Sell Songs," Reuters, June 29. http://legalminds.lp.findlaw.com/list/cyberia-l/msg45074.html

Rob, R., J. Waldfogel (2006) “Piracy on the Silver Screen,” NBER Working Paper 12010, National Bureau of Economic Research Inc.

Romer, P. (2002) “When Should We Use Intellectual Property Rights?” The American Economic Review, 92: 213-216.

Rosenthal, H., T.A. Romer (1983) "Constitution for Solving Asymmetric Externality Games,” American Journal of Political Science, 27: 1-26. 
Saijo, T., T. Yamato (1997) "Fundamental Difficulties in the Provision of Public Goods: A Solution to the Free-Rider Problem Twenty Years After," Working Paper, Osaka University.

Samuelson, P. (1954) “The Pure Theory of Public Expenditure," The Review of Economics and Statistics, 36: 387-389.

Samuelson, P. (1955) “A Diagrammatic Exposition of a Theory of Public Expenditure,” The Review of Economics and Statistics, 37: 350-356.

Samuelson, P. (1969) "Contrast between Welfare Conditions for Joint Supply and for Public Goods,” The Review of Economics and Statistics, 51: 26-30.

Sandler, T., J. Tschirhart (1980) “The Economic Theory of Clubs: An Evaluative Survey,” Journal of Economic Literature, 18: 1481-1521.

Schonfeld. E. (2002) “Peer-to-Peer Technology Goes Legit,” Business 2.0, November 22.

Singh, S., S. Ramabhadran, R. Baboescu, A.C. Snoeren (2003) "The Case for Service Provider Deployment of Super-Peers in Peer-to-Peer Networks," Working Paper, Workshop on Economics of Peer to Peer Network, University of California, San Diego.

Smith, M., R. Telang (2006) "Competing with Free: The Impact of Movie Broadcasts on DVD Sales and Internet Piracy,” Working Paper, Carnegie Mellon University.

Spencer, E. (2000) “Napster’s Shawn Fanning: The Teen Who Woke Up Web Music," Business Week, April 12.

Tamilmani, K., V. Pai, A. Mohr (2004) “SWIFT: A System With Incentives For Trading,” Working Paper, Second Workshop on Economics of Peer to Peer Network, Stony Brook University.

Thompson, C. (2005) “The BitTorrent Effect,” Wired Magazine, Issue 31.01, January. http://www.wired.com/wired/archive/13.01/bittorrent.html

van de Kragt, A., J. Orbell, R.M. Dawes (1983) "The Minimal Contribution Set as a Solution to Public Goods Problems,” American Political Science Review, 77: 112-122.

Varian, H.R. (1989) “A Solution to the Problem of Externalities When Agents Are WellInformed,” Technical Report, University of Michigan, Ann Arbor.

Varian, H.R. (1994) "Sequential Provision of Public Goods," Journal of Public Economics, 53: 165-86.

Vishnumurthy, V., S. Chandrakumar, E.G. Sirer (2003) "KARMA: A Secure Economic Framework for P2P Resource Sharing," Working Paper, Workshop on Economics of Peer to Peer Network, Cornell University. 
Walsh, K., E.G. Sirer (2005) “Thwarting P2P Pollution Using Object Reputation,” Technical Report TR2005-1980, Computer Science Department, Cornell University.

Wang, Y, J. Vassileva (2003) "Trust and Reputation Model in Peer-to-Peer Networks," Proceedings of the Third International Conference on Peer-to-Peer Computing (P2P'03), IEEE Computer Society.

Young, K.S., B.J. Thomas, T.B. Pahl, M.K. Engle (2005) "Peer-to-Peer File-Sharing Technology: Consumer Protection and Competition Issues," Staff Report, Federal Trade Commission, June. 